\title{
Multi-stable mechanism of an oscillating-body wave energy converter
}

\author{
Liang Li, Xiantao Zhang, Zhiming Yuan, Yan Gao
}

\begin{abstract}
The present research aims to utilize the multi-stable mechanism to increase the energy conversion of the oscillatingbody wave energy converter (WEC). Considering that a majority of WEC concepts are linear and single-stable, a nonlinear springdamper model is proposed to achieve the multi-stable mechanism. The system is either monostable or bistable depending on the initial condition of the springs. The energy conversion of a heaving point-absorber with the nonlinear spring-damper model is investigated in the present research. Firstly, a state-space dynamic model of the heaving point-absorber is developed. Then the energy conversion at both the monostable mode and the bistable mode is simulated. The present research also illustrates the response feature at the two stable modes. It is found the nonlinear pointabsorber behaves like a linear system in the monostable mode. The energy conversion is just increased slightly. Nevertheless, the energy absorption is increased substantially in the bistable mode. It is found that the velocity phase is adjusted in the bistable mode, leading to the enhancement of the energy conversion.
\end{abstract}

Index Terms - wave energy converter; multi-stable mechanism; power take-off system; renewable energy; energy absorption.

\section{INTRODUCTION}

$\mathrm{G}$ LOBAL demand for energy is expected to climb considerably and the world is pursuing economic and sustainable energy sources to keep up with this considerable demand growth. Compared with other ocean energy resources, such as wind and tide, wave energy is a kind of resource with high power density and all-day availability. Owing to these advantages, wave energy is regarded as a prospective solution to the generation of sustainable energy. To harvest energy from the ocean waves, wave energy converters (WECs) with various energy conversion mechanisms are developed. Babarit et al. [1] proposed a fixed-bottom WEC which was driven by the pressure-differential. Ghasemi et al. [2] developed a nonlinear model of a bottom-hinged flap device. Hsieh et al. [3] developed an oscillating water column WEC with double chambers. Compared with other categories of WECs, the oscillating-body WEC possesses the advantage of a simple energy conversion mechanism, which converts the relative motion with respect to a fixed or floating reference into electric power. Typical, a hydraulic motor or a direct drive generator is used to convert the wave energy. In this circumstance, the PTO system can be approximated as a linear spring-damper system.

It is well-known that a linear oscillation system is sensitive

L. Li, Z.M. Yuan (corresponding author), and Y. Gao, are with Department of Naval Architecture, Ocean and Marine Engineering, University of Strathclyde, (e-mail: liang.li@strath.ac.uk; zhiming.yuan@strath.ac.uk; yan.gao@strath.ac.uk). to the frequency of the excitation, and thereby the efficiency of an oscillating-body WEC is unsatisfactory in random ocean waves, which consist of multiple frequency components [4]. In addition to increasing the energy conversion using the control [5-7], the nonlinear behavior could also be utilized. To harvest energy from the ocean waves more efficiently, various types of nonlinear PTO systems are developed. One remarkable advantage of the nonlinear system is the potential capability of energy harvesting in a broad-band random excitation [8]. Lopez et al. [9] examined the influence of PTO system configuration on the performance of the so-called CECO system under a wide range of wave conditions. Zhang and Yang [10] showed that a PTO system with nonlinear spring could harvest more energy in random waves. They all showed that the energy absorption could be enhanced with a nonlinear PTO system.

The feature of a nonlinear energy capture system can be represented by the stability mechanism. A linear system is monostable whereas a nonlinear system is multi-stable. For example, a bistable system has a double-well potential with a single unstable equilibrium position and two stable equilibrium positions. The stability mechanism was firstly studied in the field of vibration energy [11]. Panigrahi et al. [12] measured the twinkling energy with nonlinear snap-through structures experimentally. Baglio et al. [13] presented a nonlinear device for vibrational energy harvesting, which took the advantage of snap-through buckling. It is recently that the multi-stable mechanism is introduced to ocean wave energy harvesting. Younesian and Alam [14] proposed a multi-stable energy capture system, which was composed of two springs and two sliders. Zhang et al. [15] proposed an adaptive bistable power capture mechanism (composed of springs and sliders) for a point absorber WEC. They showed that this novel mechanism can both increase the frequency bandwidth and improve power capture. Xiao et al. [16] utilized the magnets to achieve the bistable mechanism of a non-linear PTO system.

Despite the proposals of various PTO configurations, most of them operate with one single stability mechanism. In this study, a nonlinear PTO system with four oblique springs is proposed, which is able to operate with multi-stable mechanism. The primary objective of the present study is to utilize the multistable mechanism to increase the energy conversion of wave energy converter. This paper is organized as following. Firstly, the configuration of the nonlinear PTO system is illustrated.

X.T. Zhang, is with Oceans Graduate School, University of Western Australia (e-mail: zhxter@outlook.com ). 
a

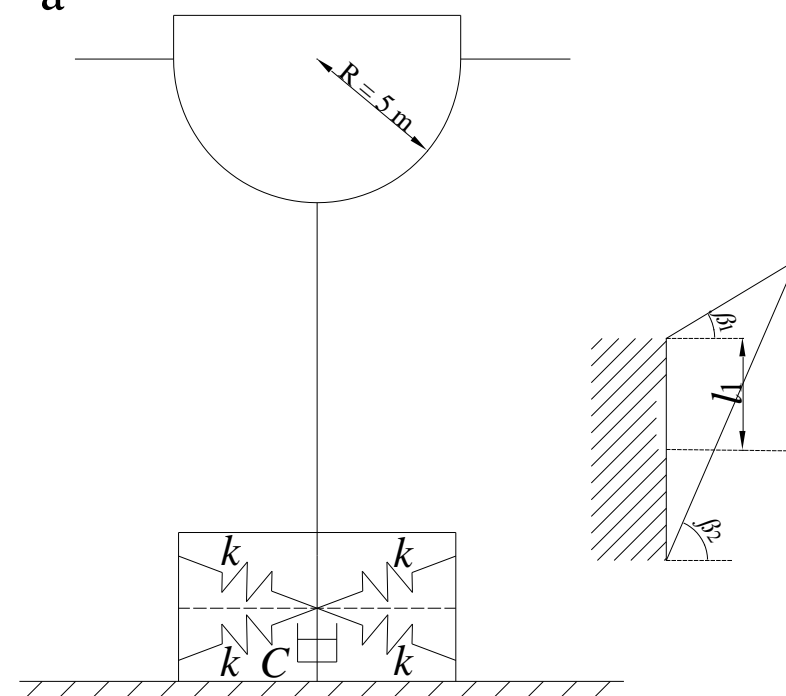

b

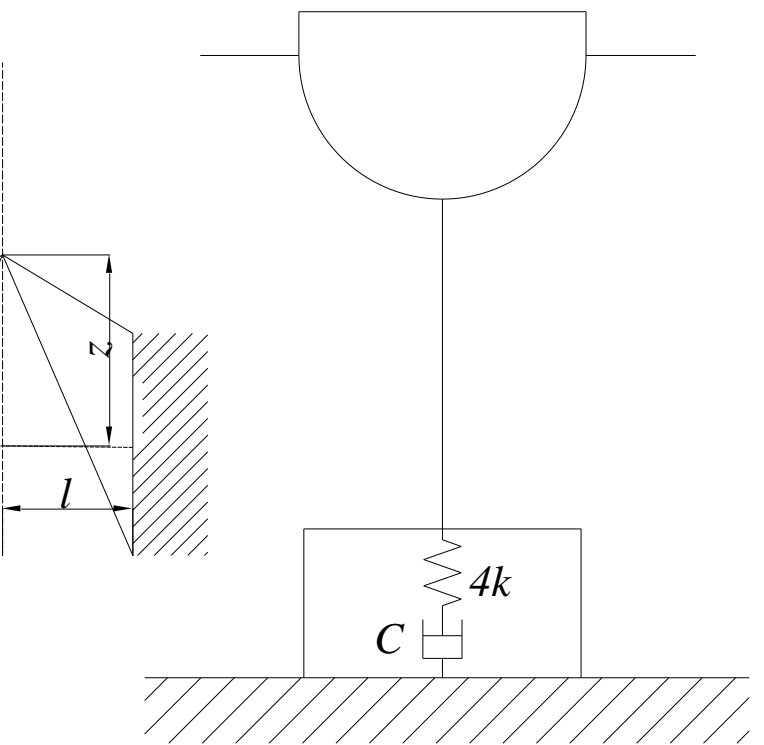

Fig. 1. The oscillating-body WEC. (a) with a nonlinear PTO system; (b) with a linear PTO system.

Then the dynamic model of a heaving point-absorber with this nonlinear system is developed. Finally, the energy conversion and the response feature of the point-absorber at different stable modes will be investigated. The main contributions of the present research are outlined as:

1. Propose a simple system that can be either monostable or bistable.

2. Clearly demonstrate the benefits of the multi-stable mechanism, in terms of energy conversion and bandwidth.

3. Show that the multi-stable mechanism increases the energy absorption by tuning the phase of the buoy velocity.

\section{NUMERICAL MODEL}

Fig. 1 shows the wave energy converter considered in the present research. The submerged buoy is a hemisphere with a radius of $5 \mathrm{~m}$. Fig. 2 shows the response amplitude operator (RAO) of the wave excitation force acting on the buoy.

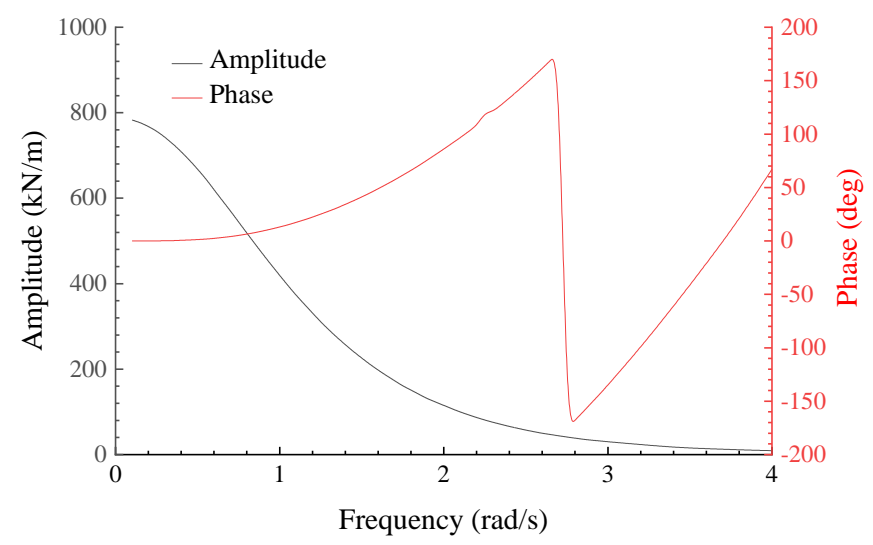

Fig. 2. RAO of the wave excitation force acting on the buoy.

The configuration of a realistic PTO system can be complicated. To focus on the scope of the present research, the PTO system is numerically treated as a spring-damper system, which is a reasonable approximation [17] and widely adopted by the other researchers [18]. The initial position of the WEC refers to $\beta_{1}=-\beta_{2}$. According to the configuration in Fig. 1 (a), the restoring force of the nonlinear PTO system is given by

$$
\begin{aligned}
& F=2 k\left(\sqrt{\left(z-l_{1}\right)^{2}+l^{2}}-l_{0}\right) \sin \beta_{1}+2 k\left(\sqrt{\left(z+l_{1}\right)^{2}+l^{2}}-l_{0}\right) \sin \beta_{2} \\
& \sin \beta_{1}=\frac{z-l_{1}}{\sqrt{\left(z-l_{1}\right)^{2}+l^{2}}}, \sin \beta_{2}=\frac{z+l_{1}}{\sqrt{\left(z+l_{1}\right)^{2}+l^{2}}}
\end{aligned}
$$

where $l_{0}$ is the initial length of the oblique springs. In this study, $l_{0}$ and $l_{1}$ are set to $4 \mathrm{~m}$ and $0.1 \mathrm{~m}$, respectively. Denote a parameter $\alpha=\sqrt{l_{1}^{2}+l^{2}} / l_{0}$ representing the initial condition of the oblique springs. The pre-tension of the oblique spring is zero at $\alpha=1$. When $\alpha>1$, the four oblique springs are stretched at $\mathrm{z}=0$. On the contrary, the springs are compressed initially. Fig. 3 displays the restoring force versus the displacement. The nonlinear PTO system has a displacement-dependent restoring stiffness. When the springs are initially stretched, the restoring force always tries to pull the float back to the equilibrium position. When $\alpha<1$, the restoring stiffness is negative within a specific range. Apparently, the PTO system is not stable within this region.

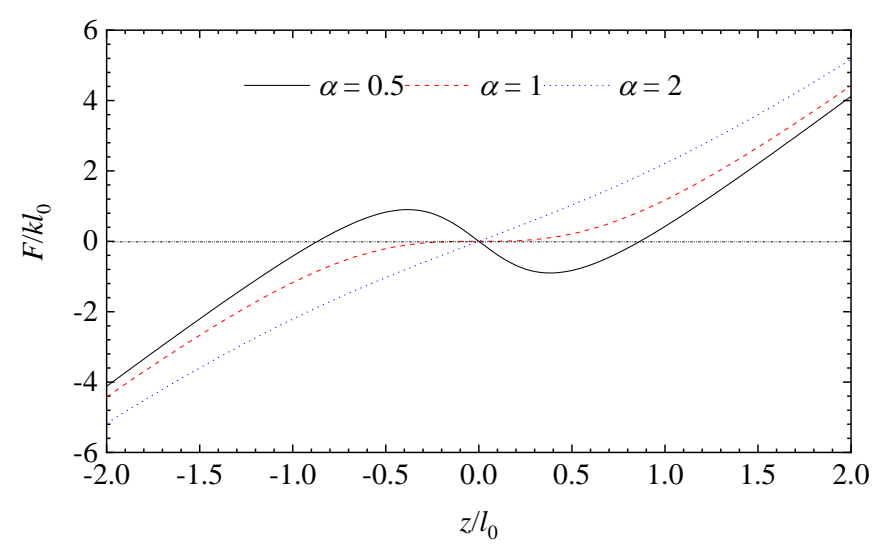

Fig. 3. Dimensionless restoring force. 
For the nonlinear PTO system, the potential energy stored in the springs is given by

$$
E=k\left[\left(\sqrt{\left(z-l_{1}\right)^{2}+l^{2}}-l_{0}\right)^{2}+\left(\sqrt{\left(z+l_{1}\right)^{2}+l^{2}}-l_{0}\right)^{2}\right]
$$

The potential energy with respect to different initial conditions of the springs is shown in Fig. 4. Depending on the initial condition of the oblique springs, the spring system is either monostable or bistable. When $\alpha=2$, the system has a single equilibrium position and a single potential well. Although the system is nonlinear, it behaves in a similar way like the linear one. There is only one type of motion for the float, which is the oscillation around the equilibrium position. At point $z=0$, the eigenvalues of the system are $0+1.21 i$ and 0 $1.21 i$ so that the system is stable. The nonlinear system is bistable with two potential wells when $\alpha=0.5$. There are three equilibrium positions. Nevertheless, $z=0$ is no longer a stable point since the eigenvalues are $1.71+0 i$ and $-1.71+0 i$. In case of any external excitation, the float will move to one of the other two stable equilibrium positions. Accordingly, there are two types of float motions. When the sea wave amplitude is small, the float is trapped within either potential well. If the wave excitation is strong enough, the float will get across the unstable position, moving around the two potential wells.

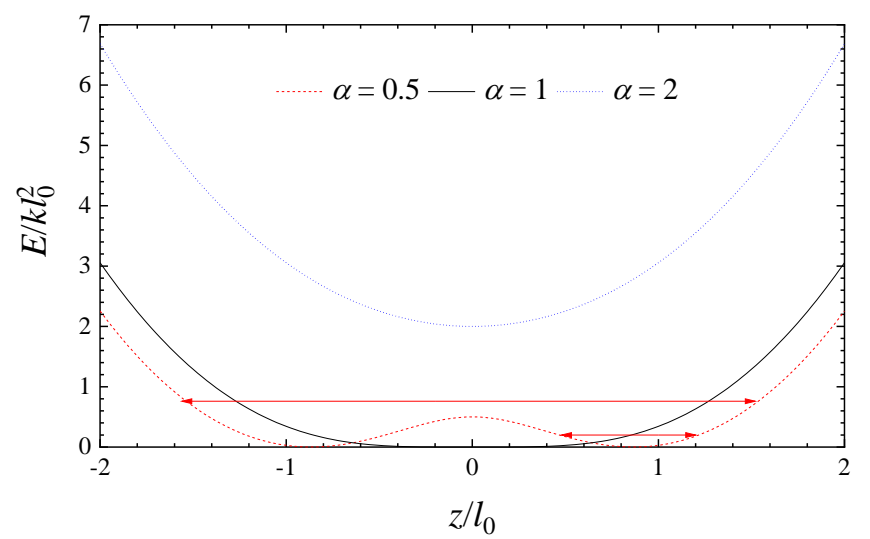

Fig. 4. Multi-stable mechanism.

A state-space representation is developed for the dynamics of the float. Based on the impulse response theory, the timedomain motion equation of the floater is given by

$$
\begin{aligned}
& (M+m) \ddot{z}(t)+\int_{0}^{t} H(t-\tau) \dot{z}(\tau) d \tau+\rho g \pi R^{2} z(t) \\
= & F_{\text {wave }}(t)-C \dot{z}(t)-2 k\left(1-\frac{l_{0}}{\sqrt{\left(z-l_{1}\right)^{2}+l^{2}}}\right)\left(z-l_{1}\right) \\
& -2 k\left(1-\frac{l_{0}}{\sqrt{\left(z+l_{1}\right)^{2}+l^{2}}}\right)\left(z+l_{1}\right)
\end{aligned}
$$

where $M$ is the mass of the floater and $m$ is the added mass at infinite frequency. $z, \dot{z}$, and $\ddot{z}$ are the displacement, the velocity, and the acceleration. $\rho$ is the water density. $g$ is the acceleration of gravity. $H$ is the so-called retardation kernel representing the memory effect of the free water surface, which can be either acquired from the added mass or the potential damping. $F_{\text {wave }}$ is the sea wave excitation force.

Denote a system with input $x(t)$ and output $y(t)$, the following three formulas are available to describe the dynamic process

$\frac{d^{n} y}{d t^{n}}+q_{n-1} \frac{d^{n-1} y}{d t^{n-1}}+\ldots+q_{0} y=p_{n-1} \frac{d^{n-1} x}{d t^{n-1}}+\ldots+p_{1} \frac{d x}{d t}+p_{0} x$

$y(t)=\int_{0}^{t} H(t-\tau) x(\tau) d \tau$

$\dot{\boldsymbol{u}}(t)=\boldsymbol{A} \cdot \boldsymbol{u}(t)+\boldsymbol{B} x(t)$

$y(t)=\boldsymbol{C} \cdot \boldsymbol{u}(t)$

Equation. (4) is an ordinary differential equation with order $n$. (5) is the convolution integral. (6) is the state-space representation, in which $\boldsymbol{u}(t)$ is the state vector with dimension $n \times 1 . \boldsymbol{A}, \boldsymbol{B}$, and $\boldsymbol{C}$ are the system matrix, the state matrix, and the output matrix, with dimension $n \times n, n \times 1$, and $1 \times n$. Combing (4) and (6), we get

$\boldsymbol{A}=\left[\begin{array}{ccccc}-q_{n-1} & -q_{n-1} & \cdots & -q_{1} & -q_{0} \\ 1 & 0 & \cdots & 0 & 0 \\ 0 & 1 & \cdots & 0 & 0 \\ \vdots & \vdots & \ddots & 0 & 0 \\ 0 & 0 & \cdots & 1 & 0\end{array}\right], \boldsymbol{B}=\left[\begin{array}{c}1 \\ 0 \\ 0 \\ \vdots \\ 0\end{array}\right]$

$\boldsymbol{C}=\left[\begin{array}{lllll}p_{n-1} & p_{n-2} & \cdots & p_{1} & p_{0}\end{array}\right]$

Combing (5) and (6), we get

$H(t)=\boldsymbol{C} e^{A t} \boldsymbol{B}$

Taking the Laplace transform of (8)

$H(s)=\frac{p_{n-1} s^{n-1}+p_{n-2} s^{n-2}+\cdots+p_{1}+p_{0}}{s^{n}+q_{n-1} s^{n-1}+\cdots+q_{1}+q_{0}}$

The frequency-domain expression of the retardation kernel $H$ is expressed as

$H(i \omega)=\lambda(\omega)+i \omega[\mu(\omega)-m]$

where is $\mu$ the added mass and $\lambda$ is the potential damping.

Equation (9) and (10) are correlated through the Fourier transformation

$\tilde{F}\{H(t)\}=H(i \omega)=\left.H(s)\right|_{s=i \omega}$

$\lambda(\omega)+i \omega[\mu(\omega)-m]=\frac{p_{n-1}(i \omega)^{n-1}+\ldots+p_{1}+p_{0}}{(i \omega)^{n}+q_{n-1}(i \omega)^{n-1}+\ldots+q_{0}}$

Denote a state vector $\boldsymbol{X}=\left[z, \dot{z}, \boldsymbol{u}^{\mathrm{T}}\right]^{\mathrm{T}}$, the motion equation of the float is re-expressed as 


$$
\begin{aligned}
& \dot{\boldsymbol{X}}=\boldsymbol{\lambda}(\boldsymbol{X})+\boldsymbol{\eta} \\
& \boldsymbol{\lambda}=\left[\begin{array}{ccc}
0 & 1 & \mathbf{0} \\
-\frac{\rho g \pi R^{2}+2 k\left(1-l_{0} / \sqrt{\left(X_{1} \pm l_{1}\right)^{2}+l^{2}}\right)}{M+m} & -\frac{C}{M+m} & -\frac{\boldsymbol{C}}{M+m} \\
\mathbf{0} & \boldsymbol{B} & \boldsymbol{A}
\end{array}\right] \\
& \boldsymbol{\eta}=\left[\begin{array}{c}
\frac{F_{\text {wave }} \pm 2 k\left(1-l_{0} / \sqrt{\left(X_{1} \mp l_{1}\right)^{2}+l^{2}}\right) l_{1}}{M+m} \\
\mathbf{0}
\end{array}\right]
\end{aligned}
$$

Equation. (12) is solved with the initial condition $\boldsymbol{X}(0)=\mathbf{0}$ using the 4-th order Runge-Kutta method. Then, the average energy absorption is given by

$$
\begin{aligned}
& P^{*}=P / \rho g A^{2} \\
& P=\frac{1}{T} \int_{0}^{T} C \dot{z}^{2}(t) d t
\end{aligned}
$$

where $A$ is the wave amplitude.

\section{Simulation Results}

As discussed, the nonlinear PTO system is either monostable or bistable depending on the initial condition of the oblique springs. This section will examine the power absorption of the nonlinear PTO system at the two stable modes, respectively. A comparison is made against the linear PTO system to elaborate whether the multi-stable mechanism can increase the energy conversion. Additionally, the response feature at the two stable modes will be demonstrated and compared with that of the linear one. A critical frequency and a critical damping of the linear PTO system are identified based on (14). In this study, $\omega_{n}=1.5 \mathrm{rad} / \mathrm{s}$ and $C_{n}=8.14 \times 10^{5} \mathrm{~kg} / \mathrm{s}$.

$$
\begin{aligned}
& \omega_{n}=\sqrt{\frac{\rho g \pi R^{2}+4 k}{M+m(\infty)}} \\
& C_{n}=2 M \omega_{n}
\end{aligned}
$$

The simulations are run in both regular and irregular waves. In the regular wave, various combinations of the wave frequency and the PTO damping coefficient are designed. For the random wave case, three sea states are considered (Table I). The random waves are represented by the JONSWAP wave spectrum and Fig. 5 plots the Bode plot of the stochastic wave forces corresponding to Case1.

Table I

RANDOM WAVE CONDITIONS

\begin{tabular}{llll}
\hline & Case1 & Case2 & Case 3 \\
\hline Hs (m) & 2 & 4 & 6 \\
$\operatorname{Tp}(\mathrm{s})$ & 11.11 & 12.33 & 13.27 \\
\hline
\end{tabular}
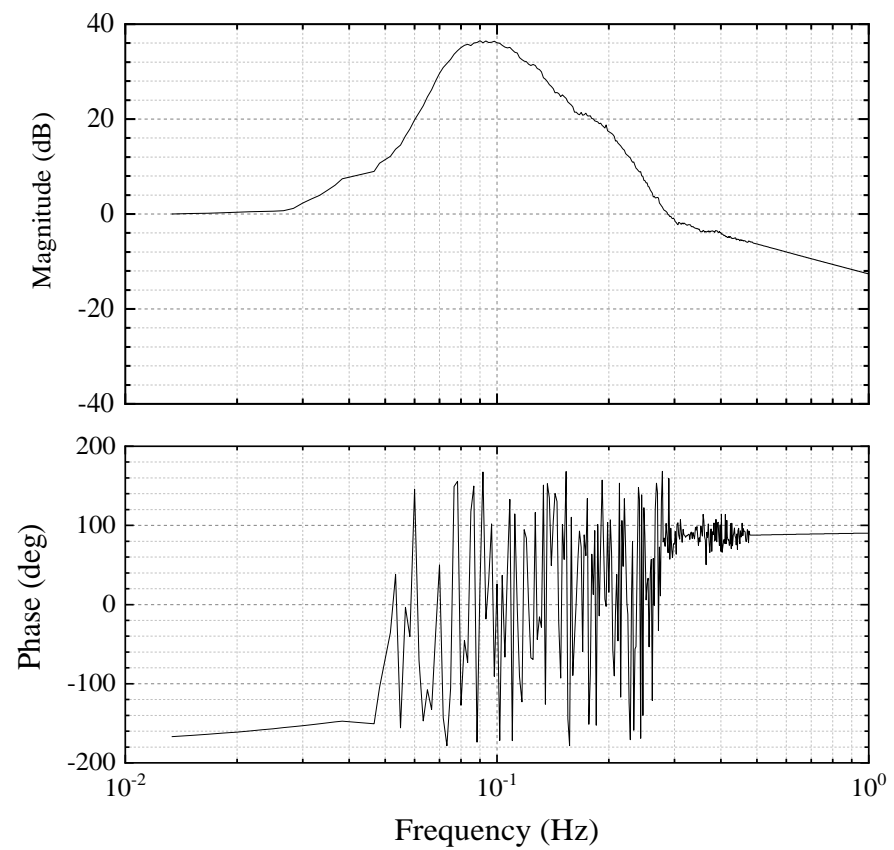

Fig. 5. Bode plot of the random wave excitation forces (Case1).

\section{A. Monostable mode $(\alpha \geq 1)$}

Fig. 6 shows the power absorption with various initial conditions of the oblique springs. The optimal frequency for the two PTO systems is nearly identical regardless of parameter $\alpha$, which is around $0.5 \cdot \omega_{n}$. The maximum power output of the linear PTO system is 8.37. Due to the nonlinear behavior of the oblique springs, this value reaches 9.40 when $\alpha=1$, increased by $12.5 \%$. When $\alpha=8$, the power capture of the nonlinear PTO system is nearly the same with that of the linear one. The sensitivity of the power production to the damping coefficient is illustrated in Fig. 7. Regardless of the initial spring condition, the energy conversion is mostly maximized at $C=C_{n}$. As parameter $\alpha$ increases, the energy absorption of the nonlinear PTO system approaches to that of the linear one.

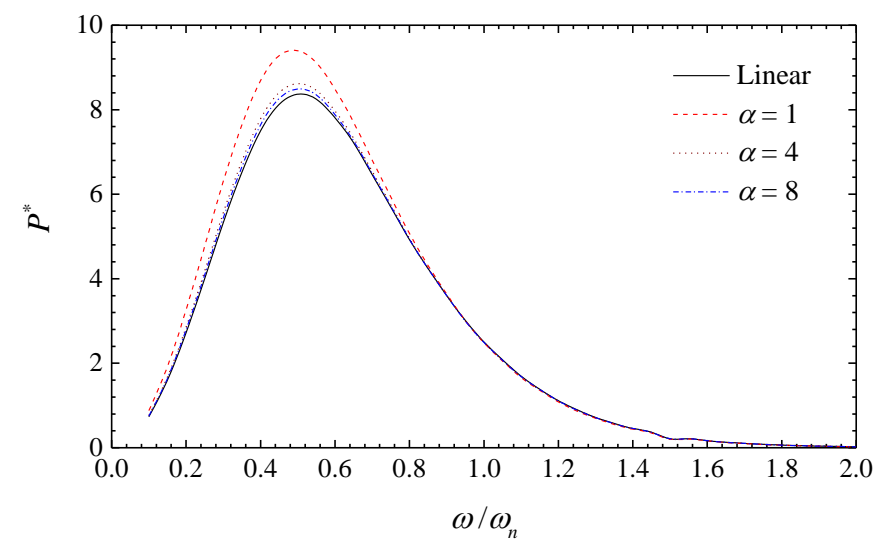

Fig. 6. Energy absorption with different wave frequencies, $A=1 \mathrm{~m}, C$ $=C_{n}$. 


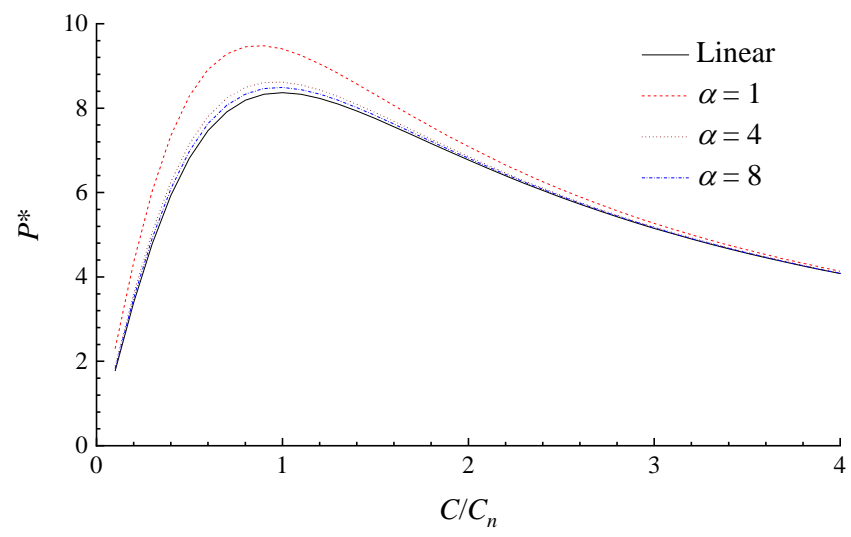

Fig. 7. Energy absorption with different damping coefficients, $A=1$ $\mathrm{m}, \omega=0.5 \cdot \omega_{n}$

The motions of the float are plotted in Fig. 8. Due to the nature of the linear PTO system, the motion is sinusoidal and thereby the phase portrait is elliptical trajectory. It is interesting to find that the motion of the nonlinear WEC is also nearly sinusoidal even if the restoring stiffness is nonlinear. The features of the motion and the phase portrait are very like those of the linear system.
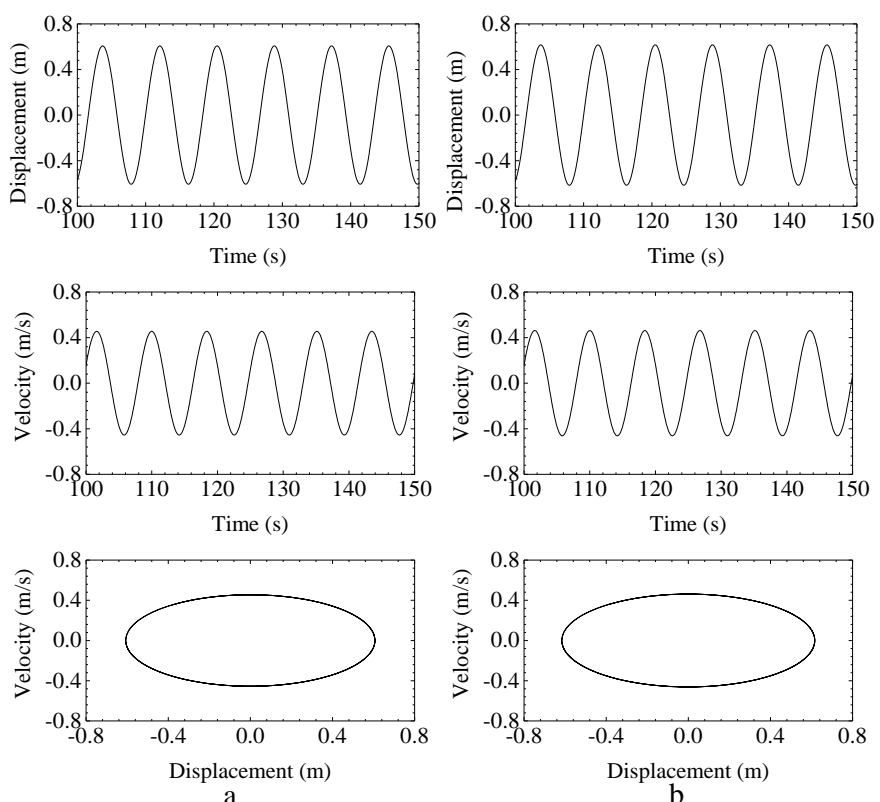

Fig. 8. Steady motion of the floater, $A=1 \mathrm{~m}, \omega=0.5 \cdot \omega_{n}$. (a) Linear PTO system; (b) Nonlinear PTO system, $\alpha=4$.

Fig. 9 demonstrates the sensitivity of the energy absorption to the wave frequency and the PTO damping coefficient. In general, the discrepancies between the linear and the nonlinear PTO systems are limited. The nonlinear PTO system increases the optimum energy absorption by no more than $14 \%$. Besides, the optimal operation conditions identified are also very close (see Fig. 10). It suggests that the power capture performances of the two WECs are very similar.
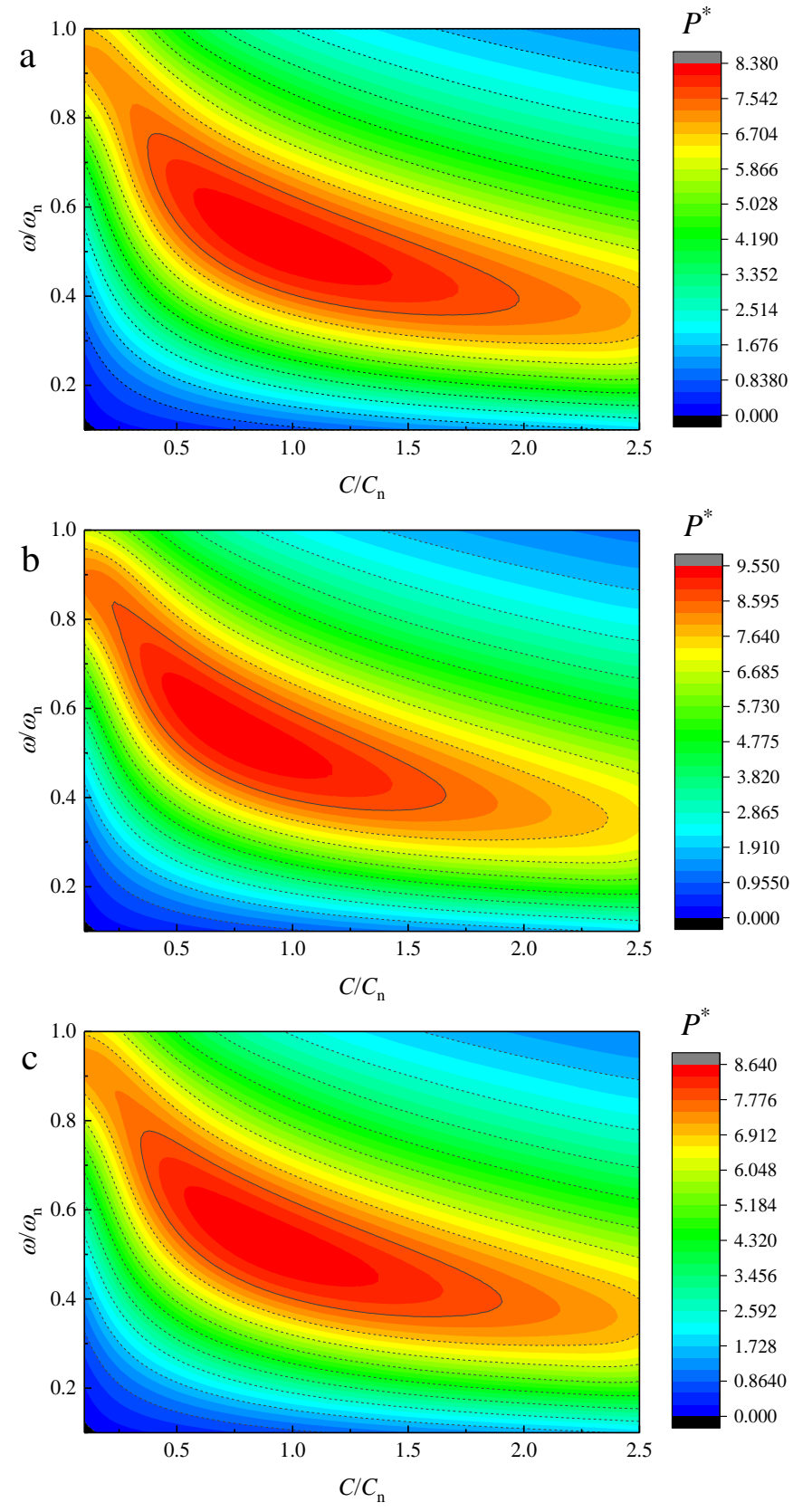

Fig. 9. Variation of energy absorption with wave frequency and PTO damping, $A=1 \mathrm{~m}$. (a) Linear; (b) Nonlinear, $\alpha=1$; (c) Nonlinear, $\alpha=$ 4.

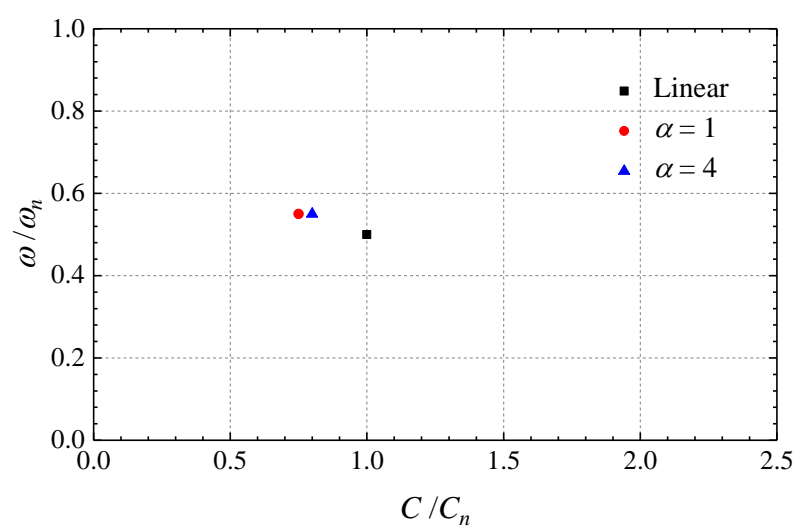

Fig. 10. Optimum operational conditions (monostable mode). 
An effective power contour (the solid inner contour in Fig. 9) is drawn to identify the region within which $P^{*}>0.9 \cdot P^{*}{ }_{\text {optimum }}$ is satisfied. Then, the damping bandwidth (the largest longitude width of the effective power contour) and the frequency bandwidth (the largest vertical width of the effective power contour) are defined, which represent the sensitivity of the energy absorption to the two variables. The damping bandwidth and the frequency bandwidth are plotted in Fig. 11. Checking the geometry of the rectangular, it suggests that both the linear and the nonlinear PTO systems are sensitive to the wave frequency detuning whereas robust to the PTO damping. The nonlinear PTO system only gives a slightly narrowed damping bandwidth than the linear system whereas the frequency bandwidth is somewhat broadened. It inherently implies that the nonlinear WEC may harvest more energy in random waves

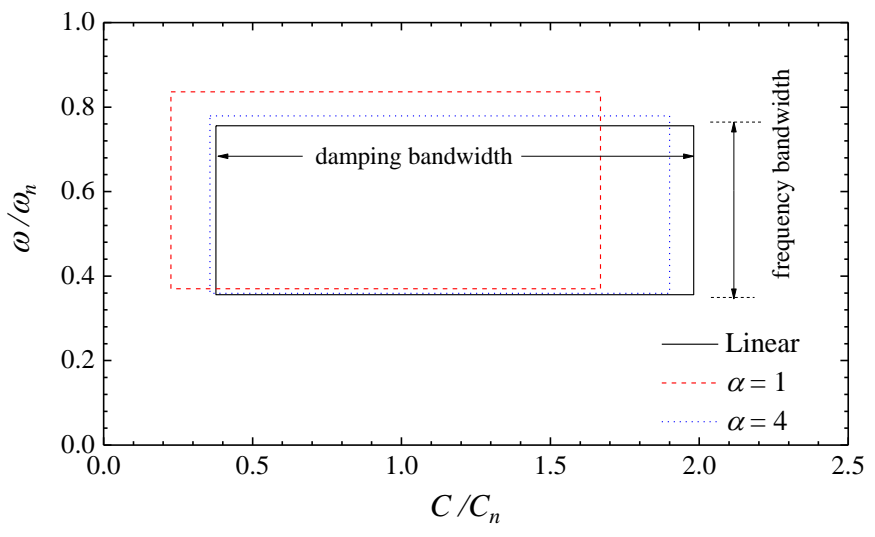

Fig. 11. Bandwidth (monostable mode).

Table II compares the total energy absorption in random waves over a 1-hour period when the two comparative PTO systems are used. As shown, the total energy absorption of the nonlinear WEC is increased by $10 \%$ approximately.

Table II 1-HOUR ENERGY ABSORPTION, $\alpha=1, C=C_{n}$.

\begin{tabular}{llll}
\hline & Case1 & Case 2 & Case3 \\
\hline Linear & $37 \mathrm{~kW} \cdot \mathrm{h}$ & $142 \mathrm{~kW} \cdot \mathrm{h}$ & $303 \mathrm{~kW} \cdot \mathrm{h}$ \\
Nonlinear & $42 \mathrm{~kW} \cdot \mathrm{h}$ & $162 \mathrm{~kW} \cdot \mathrm{h}$ & $346 \mathrm{~kW} \cdot \mathrm{h}$ \\
\hline
\end{tabular}

According to the above results, although the nonlinear PTO system is able to enhance the energy efficiency at the monostable mode, it generally behaves like the linear system. To interpret why the nonlinear WEC behaves like the linear one, we expand the restoring force at $z=0$ as Taylor series

$$
\begin{aligned}
F(z) & =4 k z+12 k l^{2} l_{0}\left[\frac{1}{\sqrt{\left(l_{1}^{2}+l^{2}\right)^{5}}}-\frac{5 l_{1}^{2}}{\sqrt{\left(l_{1}^{2}+l^{2}\right)^{7}}}\right] z^{3}+O\left(z^{5}\right) \\
& =K_{1} z+K_{2} z^{3}+O\left(z^{5}\right)
\end{aligned}
$$

where the second term represents the nonlinear effect. When $\alpha$ $=8, K_{2} / K_{1}$ is as low as 0.003 . It indicates that the nonlinear component is nearly negligible compared with the linear component. Therefore, the nonlinear system behaves very like the linear one.

\section{B. Bistable model $(\alpha<1)$}

Fig. 12 demonstrates how parameter $\alpha$ influences the energy absorption when the PTO damping coefficient is fixed at $C_{n}$. The dimensionless power production increases significantly as $\alpha$ becomes smaller, especially within the low-frequency range. At the same time, the optimal frequency keeps shifting to the left side. This is because the bistable mechanism produces extra negative stiffness to the system, which reduces the resonance frequency. Fig. 13 displays how the energy absorption varies with the damping coefficient. Similarly, the energy conversion is increased substantially when parameter $\alpha$ decreases. Additionally, the optimal damping coefficient shifts to the lowlevel region.

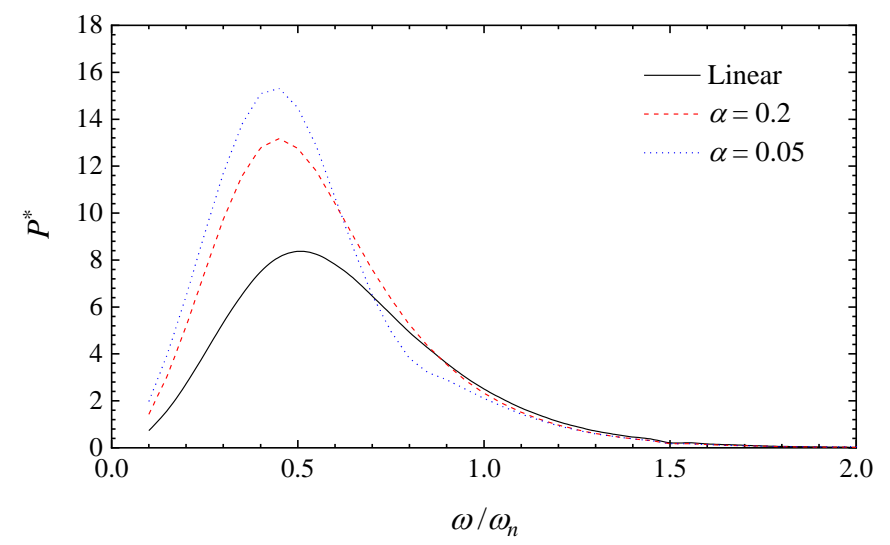

Fig. 12. Energy absorption at different wave frequencies, $A=1 \mathrm{~m}, C=$ $C_{n}$.

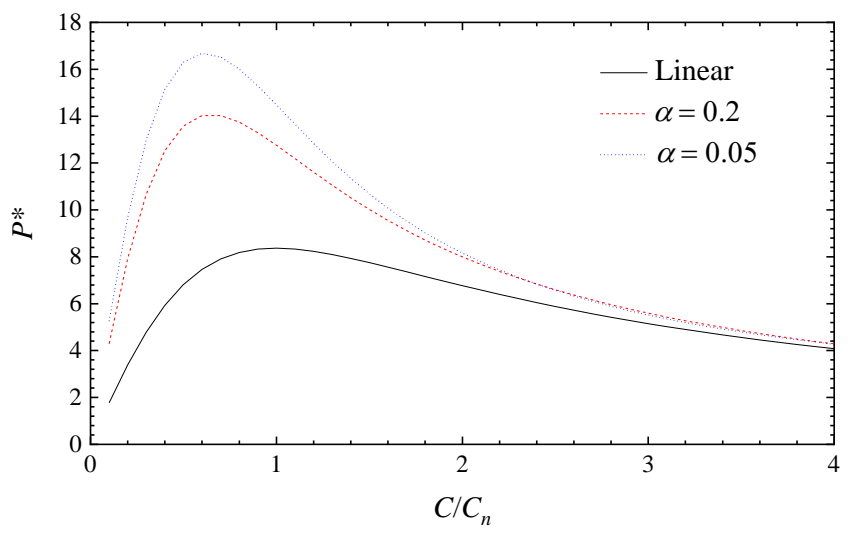

Fig. 13. Energy absorption with different damping coefficients, $A=1$ $\mathrm{m}, \omega=0.5 \cdot \omega_{n}$.

The variation of the energy absorption with respect to the wave frequency and the PTO damping is shown in Fig. 14. The three contours exhibit notable discrepancies, suggesting that the nonlinear PTO system is very sensitive to $\alpha$. The optimum energy absorption increases from 8.4 to 15.5 when $\alpha=0.2$. This value continues increasing and reaches 19.2 when $\alpha=0.05$. Besides, the identified optimum operational conditions are far from each other (see Fig. 15). If the nonlinear PTO system can work near its optimum operational condition, the energy harvesting efficiency will be significantly improved. 

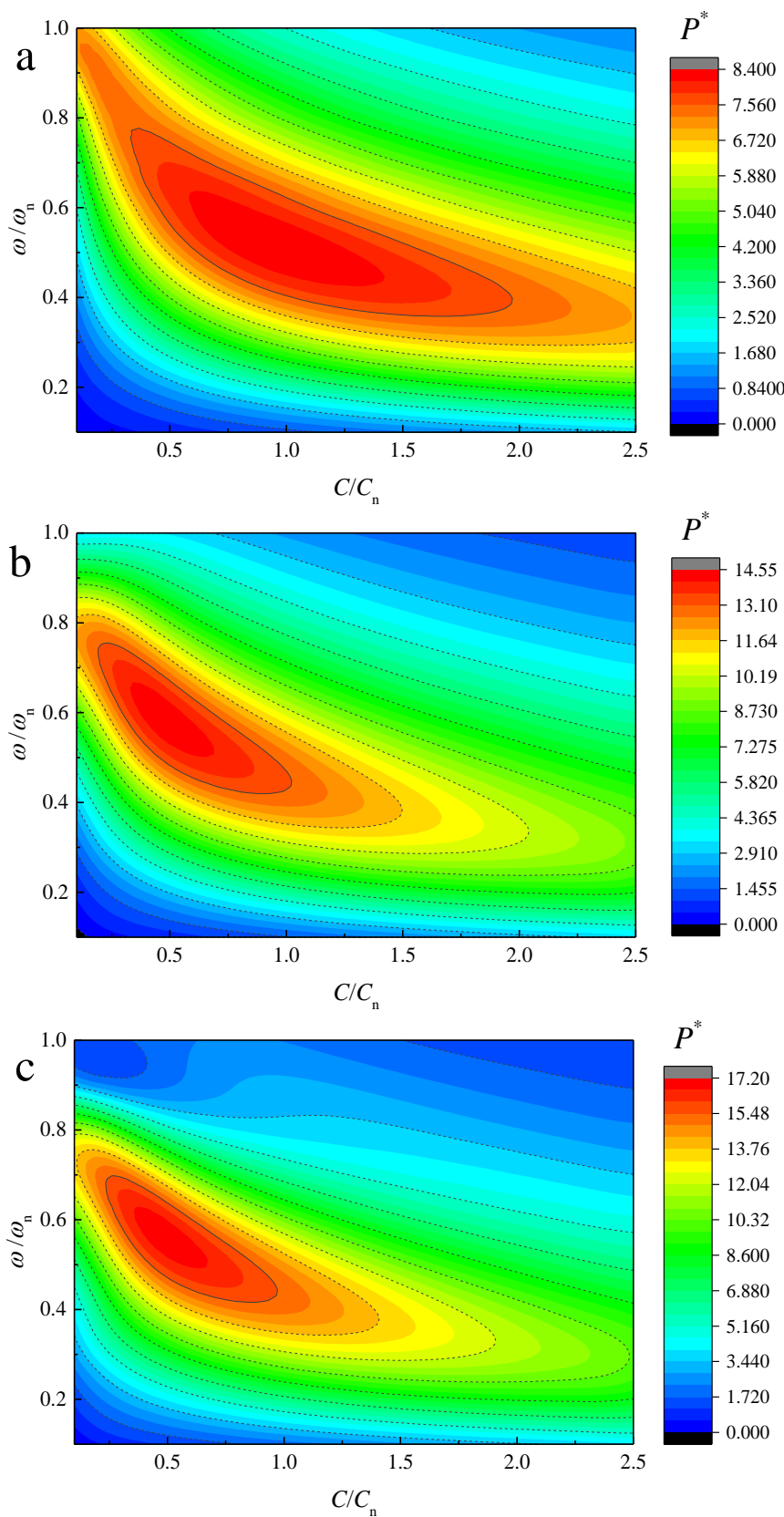

Fig. 14. Variation of energy absorption with wave frequency and PTO damping, $A=1 \mathrm{~m}$. (a) Linear; (b) Nonlinear, $\alpha=0.2$; (c) Nonlinear, $\alpha$ $=0.05$.

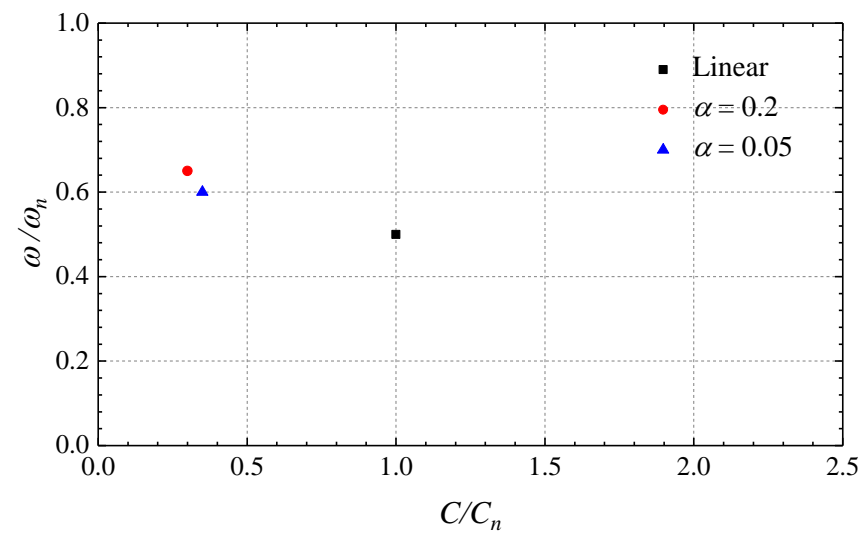

Fig. 15. Optimum operational conditions (bistable mode).
Fig. 16 displays the bandwidths of the wave frequency and the PTO damping. At the monostable mode, the energy absorption responds to the variation of frequency and damping in the same way regardless of the initial condition of the oblique springs. Nevertheless, parameter $\alpha$ has a considerable influence on the sensitivity of the energy absorption to the two parameters at the bistable mode. The optimum energy absorption increases significantly when $\alpha$ becomes smaller. At the same time, the bandwidths of frequency and damping are both narrowed implying that the WEC is more sensitive to the variation of environmental conditions. At first sight, the nonlinear PTO system is not sure to harvest more energy than the linear one. The bistable mechanism is beneficial to the optimum power production but the effective bandwidth is narrowed at the same time. Nevertheless, investigating the contours in Fig. 14 quantitatively, it is found that the nonlinear PTO system $(\alpha=$ 0.05 ) gives a larger power output than the linear one in most part of the region considered. Therefore, the nonlinear WEC still harvests more energy in random waves even if it is more sensitive to the wave frequency and the damping coefficient.

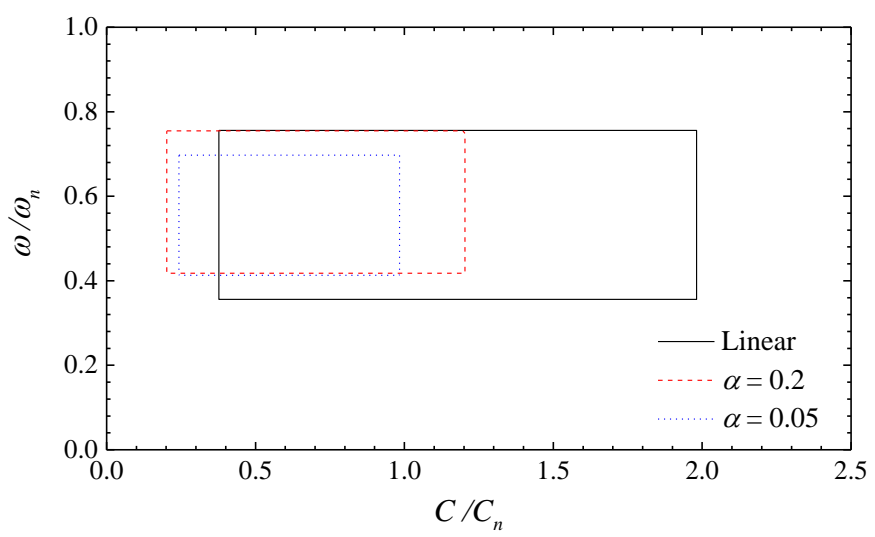

Fig. 16. Bandwidth (bistable mode).

The total energy absorbed in random waves is shown in Fig. 17. Unlike that at the monostable mode, the energy is enhanced substantially at the bistable mode.

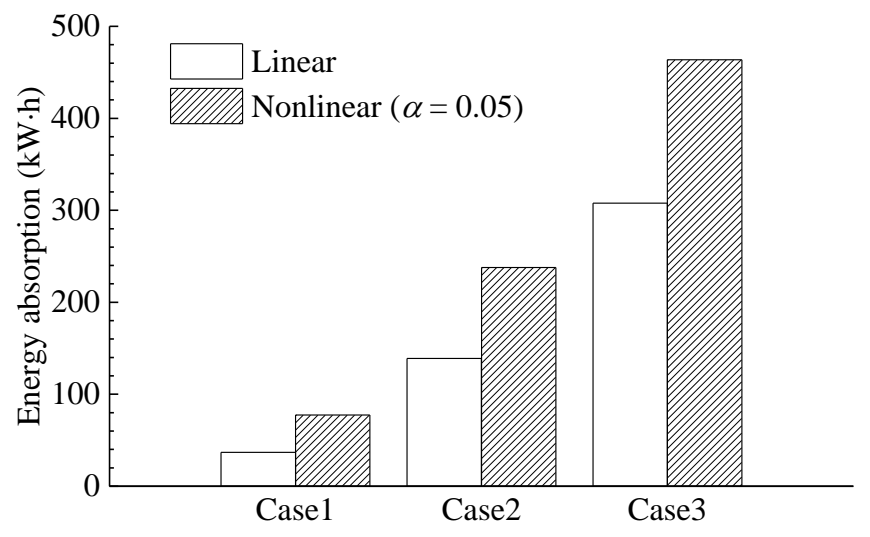

Fig. 17. Overall energy absorption during a 1-hour period, $C=C_{n}$.

One may wonder why the energy absorption is increased substantially at the bistable mode. To elaborate the philosophy behind, two operational points $\left(C / C_{n}=0.5, \omega / \omega_{n}=0.5\right)$ and $\left(C / C_{n}=2, \omega / \omega_{n}=0.8\right)$ are selected and the motions of the float 
at the two points are shown in Fig. 18. It has been shown that the float motion is nearly sinusoidal-type at the monostable mode although the PTO system is nonlinear. Due to this fact, the nonlinear PTO system behaves very like the linear one. Nevertheless, two motion patterns are identified at the bistable mode. In Fig. 18 (a), the float crosses the unstable equilibrium position $(z=0)$ and moves around the two potential wells. Although the WEC is subject to sinusoidal wave, the bistable mechanism leads to a non-monochromatic response. Thereby, the phase portrait is not an elliptical trajectory, but surrounds around the two equilibrium positions. As shown in Fig. 3, the restoring stiffness of the four oblique springs is negative within the middle region, implying that the float is subject to repulsion within this region. Once outside the unstable region, the restoring stiffness of the oblique springs becomes positive immediately. Therefore, the velocity is held for a short period rather than continues speeding up gradually. Distinguished from the response at Point 1 , the float motion at Point 2 exhibits a new feature. The most remarkable characteristic is that the float gets trapped within one single potential well. It merely oscillates around the stable equilibrium position and unable to get across the unstable equilibrium position. Meanwhile, the motion is nearly sinusoidal, verified by the elliptical trajectory of the phase portrait which surrounds a single point. We can see that the response at Point 2 is nearly identical to that of the linear PTO system despite that the float is oscillating around a new equilibrium position. It suggests that the WEC operates with different mechanisms at various combinations of wave frequency and PTO damping and thereby the energy absorption is much more sensitive to the two variables.
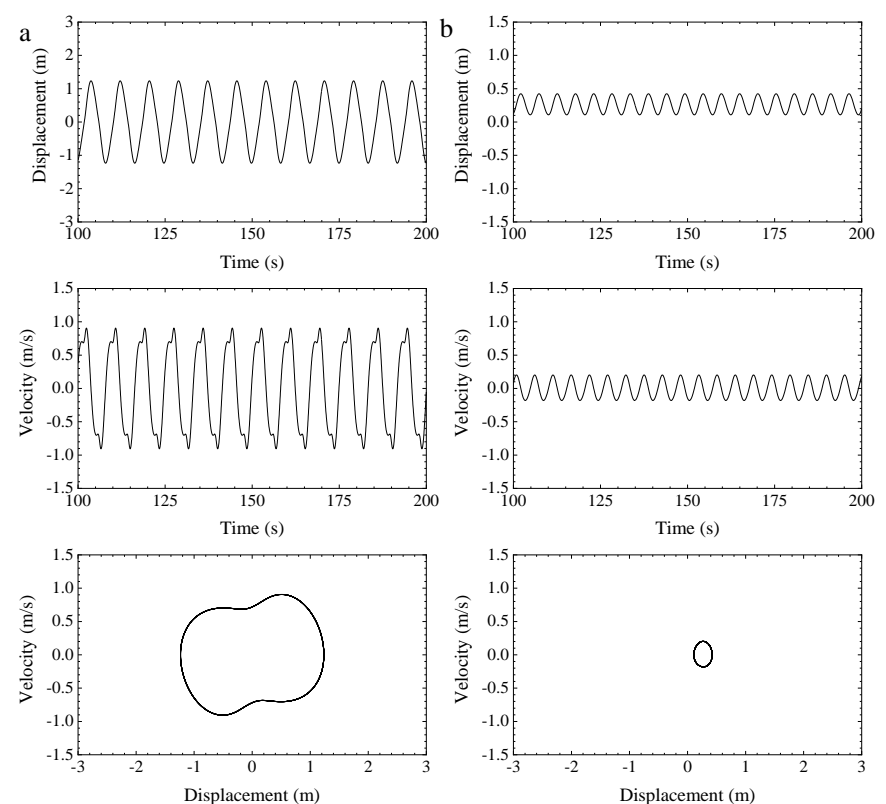

Fig. 18. Steady motion of the float, $A=1 \mathrm{~m}$. (a) Point 1; (b) Point 2.

Fig. 19 displays the phase between the float velocity and the wave excitation force. When the float experiences the unstable region, the velocity is held for a short period so that the phase of the velocity is tuned as well. This effect is very like that of a phase controller. For example, the latching control tunes the phase by holding the displacement of the float [19]. According to Budal and Falnes [20], an oscillating-body WEC harvests the most energy when the velocity is in phase with the wave force. Owing to the bistable mechanism, the velocity of the nonlinear WEC $(\alpha=0.05)$ is generally in phase with the wave force so that the energy absorption is significantly enhanced at Point 1. On the contrary, the PTO operates with a single-well potential without the unstable region at the monostable mode $(\alpha=4)$. In this circumstance, the tuning of the velocity phase is very limited so that the movement of the float is hardly changed. It becomes clear that the bistable mechanism enhances the energy absorption by tuning the velocity phase with respect to the wave excitation force.

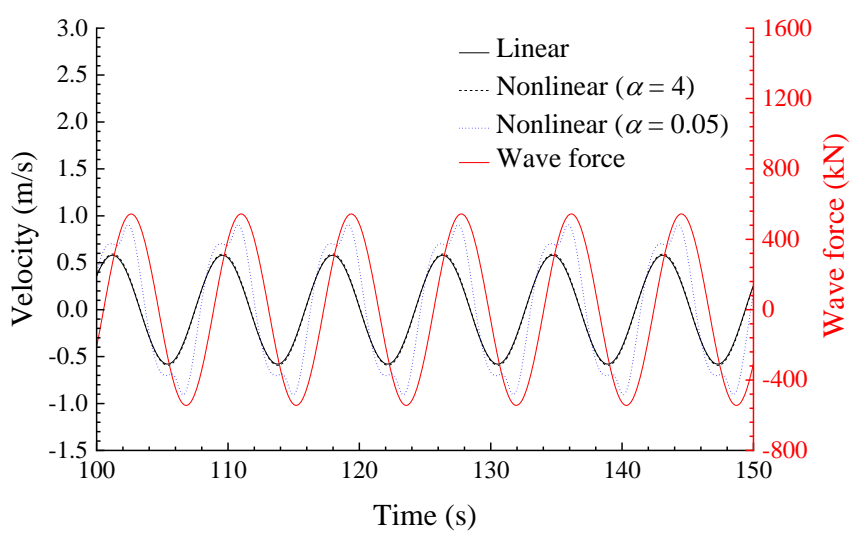

Fig. 19. Phase between velocity and wave excitation force (Point 1).

\section{CONCLUSION}

In this study, the multi-stable mechanism of an oscillatingbody WEC is investigated. Depending on the initial condition, the system can be either monostable or bistable.

The system is monostable when the springs are pre-stretched. In this circumstance, the PTO system has a single potential well with one stable equilibrium position and the restoring stiffness of the springs are always positive. Due to this nature, the nonlinear WEC behaves very like a linear one. The energy absorption is indeed increased, but just slightly. The identified optimum operation condition also remains relatively unchanged regardless of parameter $\alpha$. Besides, the bandwidths of the PTO damping and the wave frequency are hardly changed by adjusting $\alpha$. By checking the phase portrait and the time series of the float movement, it is found that the response of the float is nearly sinusoidal. In summary, the nonlinear WEC is very like to the linear one despite some tiny discrepancies.

The system is bistable when the oblique springs are precompressed. At the bistable mode, the PTO system is characteristic of a double-well potential. Accordingly, the system has an unstable equilibrium position and two stable equilibrium positions. In this circumstance, an unstable region is formed within which the restoring stiffness is negative. At the bistable mode, two types of float motions are identified. Sometimes, the float gets trapped in a single well and oscillates around the stable equilibrium position. Occasionally, the float moves around between the two potential wells on condition that the external excitations are strong enough. It explains why the WEC becomes very sensitive to the wave frequency and the PTO damping at the bistable mode. By checking the overall energy absorption under various random wave conditions, the nonlinear WEC is proved to harvest more energy. Nevertheless, 
the bandwidths of the PTO damping and the wave frequency are narrowed.

The enhancement of the energy capture is mainly attributed to the tuning of the velocity phase when the float experiences the unstable region. Simulation results show that the energy absorption is significantly increased when the velocity is in phase with the wave excitation force. Moreover, the tuning of the velocity phase is only considerable at the bistable mode when the float can move between the two potential wells. It is why the power capture is hardly improved at the monostable mode. Nevertheless, the tuning effect is not always positive. Actually, the nonlinear WEC harvests less energy at some operation conditions. A feasible solution to this problem is adjusting parameter $\alpha$.

\section{ACKNOWLEDGMENT}

The authors would like to acknowledge China Scholarship Council for the financial support (No. 201506230127).

\section{REFERENCES}

[1] A. Babarit, F. Wendt, Y. H. Yu, and J. Weber, "Investigation on the energy absorption performance of a fixed-bottom pressure-differential wave energy converter," Applied Ocean Research, vol. 65, pp. 90101, Apr 2017.

[2] A. Ghasemi, M. Anbarsooz, A. Malvandi, A. Ghasemi, and F. Hedayati, "A nonlinear computational modeling of wave energy converters: A tethered point absorber and a bottom-hinged flap device," Renewable Energy, vol. 103, pp. 774-785, Apr 2017.

[3] M. F. Hsieh, I. H. Lin, D. G. Dorrell, M. J. Hsieh, and C. C. Lin, "Development of a Wave Energy Converter Using a Two Chamber Oscillating Water Column," Ieee Transactions on Sustainable Energy, vol. 3, pp. 482497, Jul 2012.

[4] A. F. D. Falcao, "Wave energy utilization: A review of the technologies," Renewable \& Sustainable Energy Reviews, vol. 14, pp. 899-918, Apr 2010.

[5] L. Li, Z. Yuan, Y. Gao, and X. Zhang, "Wave force prediction effect on the energy absorption of a wave energy converter with real-time control," IEEE Transactions on Sustainable Energy, pp. 1-1, 2018.

[6] L. Li, Z. Yuan, and Y. Gao, "Maximization of energy absorption for a wave energy converter using the deep machine learning," Energy, vol. 165, pp. 340-349, 2018/12/15/ 2018.

[7] G. Bacelli and J. V. Ringwood, "Numerical Optimal Control of Wave Energy Converters," Ieee Transactions on Sustainable Energy, vol. 6, pp. 294-302, Apr 2015.

[8] N. Elvin and A. Erturk, Advances in energy harvesting methods: Springer Science \& Business Media, 2013.

[9] M. Lopez, F. Taveira-Pinto, and P. Rosa-Santos, "Influence of the power take-off characteristics on the performance of CECO wave energy converter," Energy, vol. 120, pp. 686-697, Feb 12017.

[10] X. T. Zhang and J. M. Yang, "Power capture performance of an oscillating-body WEC with nonlinear snap through PTO systems in irregular waves," Applied Ocean Research, vol. 52, pp. 261-273, Aug 2015.

[11] W. Y. Tseng and J. Dugundji, "Nonlinear Vibrations of a Buckled Beam under Harmonic Excitation," Journal of Applied Mechanics, vol. 38, pp. 467-476, 1971.

[12] S. R. Panigrahi, B. P. Bernard, B. F. Feeny, B. P. Mann, and A. R. Diaz, "Snap-through twinkling energy generation through frequency up-conversion," Journal of Sound and Vibration, vol. 399, pp. 216-227, Jul 7 2017.

[13] S. Baglio, V. Marletta, E. Pergolizzi, V. Ferrari, M. Ferrari, and A. R. Bulsara, "Nonlinear snap-throughbuckling devices for energy harvesting from vibrations," in Sensors, ed: Springer, 2015, pp. 409-413.

[14] D. Younesian and M. R. Alam, "Multi-stable mechanisms for high-efficiency and broadband ocean wave energy harvesting," Applied Energy, vol. 197, pp. 292-302, Jul 12017.

[15] X. Zhang, X. Tian, L. Xiao, X. Li, and L. Chen, "Application of an adaptive bistable power capture mechanism to a point absorber wave energy converter," Applied Energy, vol. 228, pp. 450-467, 2018/10/15/ 2018.

[16] X. L. Xiao, L. F. Xiao, and T. Peng, "Comparative study on power capture performance of oscillating-body wave energy converters with three novel power take-off systems," Renewable Energy, vol. 103, pp. 94-105, Apr 2017.

[17] H. Mendonca and S. Martinez, "A Resistance Emulation Approach to Optimize the Wave Energy Harvesting for a Direct Drive Point Absorber," Ieee Transactions on Sustainable Energy, vol. 7, pp. 3-11, Jan 2016.

[18] Z. Feng and E. C. Kerrigan, "Latching-Declutching Control of Wave Energy Converters Using DerivativeFree Optimization," IEEE Transactions on Sustainable Energy, vol. 6, pp. 773-780, 2015.

[19] A. Babarit and A. H. Clement, "Optimal latching control of a wave energy device in regular and irregular waves," Applied Ocean Research, vol. 28, pp. 77-91, Apr 2006.

[20] K. Budal and J. Falnes, Interacting point absorbers with controlled motion. London: Academic Press, 1980.

Liang Li received his B.S. and M.Sc. degrees from Shanghai Jiao Tong University. He is now a Ph.D. student in University of Strathclyde. His research topic is mainly relating renewable energy, with a focus on the wave energy converter control.

Xiantao Zhang received his B.S. and M.Sc. degrees from Shanghai Jiao Tong University. He is now a Ph.D. student in University of Western Australia. His main research interests include wave energy and computational fluid dynamics.

Zhiming Yuan received his B.S. and M.Sc. degrees from Jiangsu University of Science and Technology. After completing this Ph.D. study in University of Strathclyde, he stayed and worked as a lecturer until now.

Yan Gao received her B.S. and M.Sc. degrees from Shanghai Jiao Tong University. Now, she is working towards her Ph.D. degree in University of Strathclyde. Her research interest mainly focuses on renewable energy system, especially the structural integrity 